\title{
Enhancement of Laccase Production from Wood-Rotting Fungus by Co-Culture with Trichoderma longibrachiatum ${ }^{1}$
}

\author{
Hyun-Chae Jung (iD ${ }^{2, \dagger}$
}

\begin{abstract}
This work aimed to evaluate the influence of culture conditions on laccase production in the co-culture of wood-rotting fungus with Trichoderma sp. The effects of infection extent, infection time, and culture filtrate of Trichoderma sp. on the laccase production by wood-rotting fungus in co-culture were examined. T. rubrum LKY-7 and T. longibrachiatum were selected as fungi which are effective in co-culture for laccase production. A significant increase in laccase activity was observed when T. rubrum LKY-7 was co-cultured with T. longibrachiatum in glucose-peptone liquid medium, yielding an increase of more than 5 times in laccase activity, as compared with control. Laccase production by $T$. rubrum LKY-7 during co-culturing was significantly influenced by the infection extent and the infection time of $T$. longibrachiatum. Maximal laccase activity was obtained when T. rubrum LKY-7 culture was infected by T. longibrachiatum after 3 days of cultivation at an inoculum size ratio of 0.5 to 1 . The addition of culture filtrate or autoclaved mycelium of T. longibrachiatum to T. rubrum LKY-7 culture did not significantly enhance laccase production by T. rubrum LKY-7 as compared with control (mono cultures of T. rubrum LKY-7).
\end{abstract}

Keywords: laccase, co-culture, infection extent, infection time, culture filtrate

\section{INTRODUCTION}

Laccase is a blue multi copper-containing oxidase that has been found in numerous basidiomycete fungi, in various plants and insects, and even in bacteria. It has been known that laccase has many physiological functions, including lignin biosynthesis/biodegradation, stress defense, and plant pathogenesis etc. (Thurston, 1994; Cho et al., 2012; Lee et al., 2016; An et al., 2018). And due to the wide range of substrates, laccase has been shown effective in various biotechnological processes, such as pulping and bleaching, dye decolori- zation, wastewater treatment, food processing, and bioremediation of environmental pollutants (Choi et al., 2006; Hong et al., 2013; Pooja et al., 2016; ). Especially, the fact that laccase require only oxygen for catalysis has attracted much research attention for the practical use of fungal laccase in industry, unlike peroxidase. However, as the extracellular fungal laccase is produced relatively in small amounts, the use of laccase for industrial applications has been limited by low productivities in the processes (Minussi et al., 2002).

Laccase production can be enhanced by the addition of various inductive substances, including aromatic or

\footnotetext{
${ }^{1}$ Date Received December 20, 2018, Date Accepted March 6, 2019

2 Department of Forest Resources, Sunchon National University, Suncheon 57922, Republic of Korea

† Corresponding author: Hyun-Chae Jung (e-mail: jhc@sunchon.ac.kr, ORCID: 0000-0002-0063-4615)
} 
Enhancement of Laccase Production from Wood-Rotting Fungus by Co-Culture with Trichoderma longibrachiatum

phenolic compounds (Valle et al., 2015; Yadav, 2018) and metal ions (Baldrian and Gabriel, 2002; JaroszWilkolazka et al., 2004) etc. However, most of the chemical laccase inducers are environmentally toxic and expensive. Thus, many researchers have been trying to find the ways, which are safe environmentally and low-cost, to increase laccase production from white-rot fungi.

Interspecific interaction between white-rot fungi and other fungi or bacteria have shown to induce laccase activity (Savoie and Mata, 1999; Baldrian, 2004; Zhang et al., 2006; Hiscox, 2010). In interspecific fungal interaction, the extent of the increase in laccase activity could be differ, depending on the innate laccase producing ability of a species, the combination of species interacting (Iakovlev and Stenlid, 2000; Chi et al., 2007), and the types of ligninolytic enzyme secreted by each strain (Lee et al., 2017). And for high volumetric production of laccase in interspecific fungal interaction, the techniques of submerged fermentation should be applied rather than solid media. However, in submerged cultures, it is difficult to make close contact between strains in comparison with solid cultures. Flores et al. (2010) reported that the relative increase in laccase volumetric activity during TrichodermaPleurotus co-culturing in liquid media was half of that obtained when both strains were directly confronted in solid media, and laccase production in liquid media was influenced by the time of infection and agitation/ oxygenation conditions.

In previous work, we evaluated the effect of ligninolytic enzyme type secreted by each strain on ligninolytic enzyme production in interspecific interactions of wood-rotting fungi. In glucose-peptone agar medium, co-cultures of wood-rotting fungi with Trichoderma viride which do not produce any ligninolytic enzyme were very effective in the increase of laccase activity (Lee et al., 2017). The present work was to select the effective wood-rotting fungus and
Trichoderma sp. on laccase production in interspecific interaction, then to evaluate the effect of infection extent (inoculum size), infection time, and culture filtrate of Trichoderma sp. on laccase production by wood-rotting fungi in submerged co-culture.

\section{MATERIALS and METHODS}

\subsection{Fungal strains and culture conditions}

\section{Trychophyton rubrum LKY-7 (T. rubrum LKY-7)} were isolated from hardwood chip pile (Jung et al., 2001), and Pycnoporus cinnabarinus (P. cinnabarinus) were kindly supplied by Prof. Kaichang Li (Oregon State University, USA). Trichoderma harzianum, Trichoderma longibrachiatum, Trichoderma reesei, and Trichoderma viride were obtained from KCCM (Korea Culture Center of Microorganisms). These fungi were maintained on potato dextrose agar (PDA) and stored at $4^{\circ} \mathrm{C}$. For screening the effective Trichoderma sp. on laccase production by wood-rotting fungi, interspecific interactions were performed on glucose-peptone agar medium (GPA, solid medium) (30 g glucose; $10 \mathrm{~g}$ Bacto-peptone; 1.5 g KH $2 \mathrm{PO}_{4} ; 0.5$ g $\mathrm{MgSO}_{4} ; 20 \mathrm{mg}$ $\mathrm{CuSO}_{4} ; 12 \mathrm{mg} \mathrm{MnSO}_{4} ; 2 \mathrm{mg}$ thiamine-HCl; Agar 20 $\mathrm{g}$, per liter) at $\mathrm{pH}$ 5. Glucose-peptone broth medium (GPB, liquid medium) containing several glass beads (Ø $0.5 \mathrm{~mm}$ ) to avoid the formation of mycelial pellet was used for laccase production.

\subsection{Selection of effective fungi in laccase production}

In order to select effective fungi on laccase production in interspecific interaction, P. cinnabarinus or T. rubrum LKY-7 and each Trichoderma sp. were inoculated onto cellophane membranes (GelAir Cellophane, Bio-Rad 165-1779) overlaid on GPA plate at a $5 \mathrm{~cm}$ apart. The cellophane membrane was boiled twice for $15 \mathrm{~min}$ in distilled water, immersed in a flask containing distilled 
water, autoclaved for $15 \mathrm{~min}$, and then overlaid on GPA surface (Téllez-Téllez et al., 2003). As the growth of each Trichoderma sp. was faster than that of woodrotting fungi, P. cinnabarinus and T. rubrum LKY-7 were inoculated first, and Trichoderma strains were inoculated to those cultures 3 days later, respectively. One mycelial plug (mycelium of $8 \mathrm{~mm}$ diameter) was aseptically scraped from the contact zone of T. rubrum LKY-7 or $P$. cinnabarinus region in the co-cultures at days 5 and 12 of cultivation after initial contact. Laccase activities were determined from the harvested mycelium according to a previously described method (Lee et al., 2017). The self-paired culture of each wood-rotting fungus was used as control.

\subsection{Laccase production in submerged co-culture}

GPB was used for laccase production by interspecific interaction. Wood-rotting fungus and Trichoderma sp. selected from above experiments were pre-cultivated on cellophane membrane overlaid on GPA plate for 7 and 3 days, respectively. The mycelium of 6-10 mm diameter was scraped from actively growing mycelial edge on the surface of cellophane membrane and used for inoculum of each strain. Basically, first, the inoculum of wood-rotting fungus was homogenized with portable mill in $25 \mathrm{ml}$ of GPB and incubated for 3 days at $27^{\circ} \mathrm{C}$ with agitation at rate of $150 \mathrm{rpm}$. Then, the mycelium scraped from Trichoderma sp. (non-sporulating mycelium, 3 days old) was homogenized in $25 \mathrm{ml} \mathrm{GPB}$, and added into the 3 days old culture of wood-rotting fungus.

In order to evaluate the effect of infection extent of Trichoderma sp. on laccase production by woodrotting fungus, the inoculum size ratio of Trichoderma sp. to wood-rotting fungus was adjusted to 0.25 to 1 , 0.5 to 1,1 to 1 , and 2 to 1 using cork borers, then cocultivations were carried out according to the above procedure. Also, to assess the effect of infection time, the homogenized inoculums of Trichoderma sp. with inoculum size ratio of 0.5 to 1 and 1 to 1 were added into wood-rotting fungus cultures incubated for 1,3 , and 5 days, respectively. And, to estimate the effect of culture filtrate of Trichoderma sp., only Trichoderma sp. which homogenized in $25 \mathrm{ml}$ of GPB with inoculum size ratio of 0.5 to 1 were incubated for 4 days. Then, these cultures were separated with mycelium and culture filtrate through a sterile 0.2 um Millipore filter. The separated mycelium was washed thoroughly with distilled water, homogenized in $25 \mathrm{ml}$ of GPB, and autoclaved at $121{ }^{\circ} \mathrm{C}$ for $20 \mathrm{~min}$. The autoclaved mycelium and the culture filtrate were each added into 3 days old cultures of wood-rotting fungus.

All cultures described above were incubated under shaking condition at a rate of $150 \mathrm{rpm}$ at $27^{\circ} \mathrm{C}$ for 15 days. For the assay of laccase activity, $200 \mu \mathrm{l}$ culture fluid was extracted periodically in all cultures and replaced by $200 \mu$ flesh culture medium. The monoculture of wood-rotting fungus was used as control. All experiments were performed in triplicate.

\subsection{Measurement of laccase activity}

Laccase activity was determined spectrophotometrically by measuring the oxidation of 2,2' -azino-bis(3ethylbenzothiazoline-6-sulfonnic acid) (ABTS) in sodium tartrate buffer (50 mM, pH 4.5) at $420 \mathrm{~nm}$ $\left(\varepsilon \max =3.6 \times 10^{4} \mathrm{M}^{-1} \mathrm{~cm}^{-1}\right)$. One unit $(\mathrm{U})$ of laccase activity was defined as the amount of enzyme catalyzing the production of $1 \mu \mathrm{mol}$ oxidized product per $\mathrm{ml}$ of enzyme solution per minute under the assay conditions (Eggert et al., 1996).

\subsection{Native polyacrylamide gel electrophoresis (PAGE)}

For native PAGE, extracellular enzyme extracts were obtained from mycelia (solid media, GPA) and culture fluids (liquid media, GPB) after 12 days of cultivation, respectively. The scraped mycelia from self-paired 
culture and co-culture on GPA were transferred into a $1.5 \mathrm{ml}$ Eppendorf tube, to which $0.5 \mathrm{ml}$ of $50 \mathrm{mM}$ sodium tartrate buffer ( $\mathrm{pH} 4.5$ ) was added. Tubes were shaken gently and centrifuged. The filtrates were used as extracellular enzyme samples. And the enzyme extracts in liquid media (GPB) were prepared by centrifugation and filteration (0.2 um Millipore filter) after filtering the culture.

Native PAGE was performed on $10 \%$ MiniPROTEIN TGX gel (BioRad, USA) with running conditions of $35 \mathrm{~mA}$ at a constant voltage of $200 \mathrm{~V}$. The amounts of enzyme extract were adjusted to load approximately equivalent laccase activities in each well. For laccase isozyme staining, gel was immersed in $\mathbf{5 0}$ $\mathrm{mM}$ sodium tartrate buffer ( $\mathrm{pH}$ 4.5) containing $5 \mathrm{mM}$ guaiacol, and incubated at room temperature until laccase activity bands appeared.

\section{RESULTS and DISCUSSION}

\subsection{Selection of effective fungi on laccase production}

In previous work, it showed that laccase production was significantly enhanced in the co-cultures of woodrotting fungi with $T$. viride which doesn't secrete laccase on GPA when compared with other fungi which secrete laccase (Lee et al., 2017). No laccase activities were detected in mono-cultures of four Trichoderma strains used in this work (results not shown). Screening strategy for biological induction of laccase production has been generally based in the confrontation of basidiomycetes with other strains and measuring laccase activity in interaction zone (Iakovlev and Stenlid, 2000; Baldrian, 2004; Chi et al., 2007). These four strains of Trichoderma were subjected to interspecific interaction with $P$. cinnabarinus or $T$. rubrum LKY-7 on cellophane membrane of GPA plate. As shown in Table 1, significant increases of laccase activity were observed when wood-rotting fungi were confronted with Trichoderma strains. The highest activity of laccase was obtained when T. rubrum LKY-7 was co-cultured with $T$. longibrachiatum on GPA, yielding more than about 8 times increase in laccase activity, as compared with control. It has well known that laccase production of white-rot fungi is effectively enhanced by co-culturing with Trichoderma sp. (Baldrian, 2004; Zhang et al., 2006; Velázquez-Cedeño et al., 2007; Flores et al., 2009; 2010). However, the levels of activity in these studies were relatively low when compared with the results of this experiment, although there are large increases in the laccase activities in interspecific interaction.

In self-paired culture (control) of $P$. cinnabarinus or T. rubrum LKY-7, the laccase activity at day 5 of

Table 1. Laccase activities of wood-rotting fungi during co-culturing with Trichoderma strains in GPA

\begin{tabular}{|c|c|c|c|c|}
\hline \multirow{3}{*}{ Strain } & \multicolumn{4}{|c|}{ Laccase activity $(\mathrm{U} / \mathrm{ml})^{\mathrm{a}}$} \\
\hline & \multicolumn{2}{|c|}{ P. cinnabarinus } & \multicolumn{2}{|c|}{ T. rubrum LKY-7 } \\
\hline & 5 days $^{b}$ & 12 days & 5 days & 12 days \\
\hline Control $^{\mathrm{c}}$ & $0.98 \pm 0.23$ & $0.17 \pm 0.06$ & $1.45 \pm 0.24$ & $0.75 \pm 0.17$ \\
\hline T. harzianum & $1.67 \pm 0.21$ & $1.79 \pm 0.39$ & $2.48 \pm 0.36$ & $2.25 \pm 0.49$ \\
\hline T. longibrachiatum & $3.26 \pm 0.47$ & $5.02 \pm 0.43$ & $5.32 \pm 0.72$ & $8.02 \pm 0.75$ \\
\hline T. reesi & $2.15 \pm 0.26$ & $2.42 \pm 0.62$ & $2.77 \pm 0.49$ & $3.31 \pm 0.28$ \\
\hline T. viride & $5.18 \pm 0.74$ & $5.31 \pm 0.66$ & $4.19 \pm 0.32$ & $4.76 \pm 0.69$ \\
\hline
\end{tabular}

${ }^{a}$ Laccase activity per $\mathrm{ml}$ of enzyme solution extracted from the ground mycelium in $50 \mathrm{mM}$ sodium tartrate buffer

${ }^{\mathrm{b}}$ Incubation day after initial contact

' Self-paired culture of P. cinnabarinus or T. rubrum LKY-7 
cultivation decreased significantly at day 12 . However, in the co-cultures, the stability of laccase activities were improved with compared to control, rather showing the increase of laccase activities at day 12 of cultivation. Especially, in the co-culture of T. rubrum LKY-7 with T. longibrachiatum, the maximal laccase activity was remained almost constant until day 15 of cultivation (data not shown). These results are in agreement with the study of Flores et al. (2010), who has shown that the stability of laccase activity is improved during Trichoderma-Pleurotus interaction, yielding twice the activity if compared with control treatments (Trichoderma or Pleurotus alone). Also, Zhang et al. (2006) reported that the laccase activity decreases sharply in chemical induction within 2-3 days after peak, whereas it maintains $60-70 \%$ of its highest activity for at least 20 days during the co-cultivation process of Trametes sp. and Trichoderma strain. Based on these results, it was confirmed that the co-culture of T. rubrum LKY-7 with $T$. longibrachiatum has a great potential for laccase production.

\subsection{Effect of the infection extent of T. Iongibrachiatum on laccase production}

In submerged co-culture, laccase production could be influenced by the inoculum amount of each strain, unlike solid culture. The mycelial suspensions of $T$. longibrachiatum adjusted the inoculum size ratio were added into 3 days old cultures of T. rubrum LKY-7 to evaluate the effect of infection extent on laccase production. As shown in Fig. 1, T. rubrum LKY-7 produced relatively high activity of laccase when cultivated on GPB (control), and its maximum activity maintained almost the same until the end of cultivation, unlike on GPA. The laccase productions in co-cultures were clearly divided into two levels according to the inoculum size ratio. When the inoculum size ratio of Trichoderma sp. to T. rubrum LKY-7 were adjusted

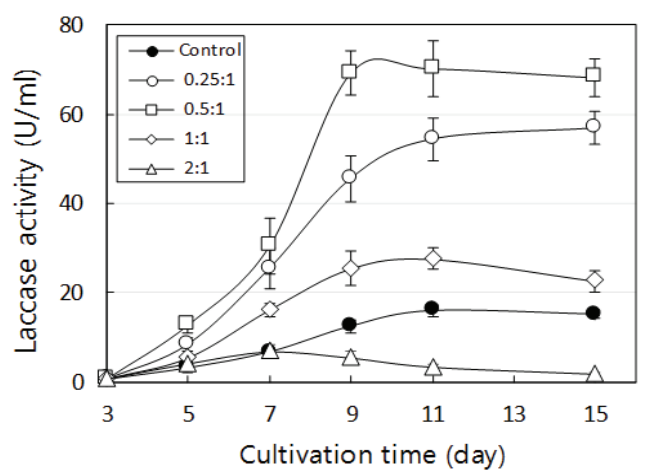

Fig. 1. Effect of the infection extent of $T$. longibrachiatum on laccase production by $T$. rubrum LKY-7.

to 0.25 to 1 or 0.5 to 1 , the laccase activities began to increase on days 5 of cultivation, then increased to 54.3 and $70.2 \mathrm{U} / \mathrm{ml}$ on days 11 , respectively. These laccase activities remained relatively stable until the end of cultivation. It suggested that the laccase activity can be induced strongly when the growth $T$. rubrum LKY-7 has a predominance in co-culture (ratio of 0.25 to 1 or 0.5 to 1 ). In contrast, when adjusted the inoculum size ratio to 1 to 1 , the laccase activities of $T$. rubrum LKY-7 significantly decreased, in comparison with that of a ratio of 0.25 to 1 or 0.5 to 1 . Especially, at the ratio of 2 to 1 , no induction effect on the laccase activity was observed, which indicated that when the infection extent of $T$. longibrachiatum is beyond a certain amount so that its growth is overgrown in co-culture, the laccase production by T. rubrum LKY-7 could be suppressed and inhibited. These results are in agreement with Velázquez-Cedeño et al. (2007), who reported that the laccase production in interspecific interaction of Pleurotus ostreatus with $T$. longibrachiatum seems to depend on the relative ability of the two fungi to colonize liquid or solid medium. Cupul et al. (2014) reported that the low inoculum amount enhances laccase activity by $T$. maxima in T. maxima- $P$. carneus co-cultures, and the amount of mycelial disks used can affect enzyme activity in co-cultures. 
On the whole, the inoculations in submerged culture are performed with the addition of homogenized mycelium, fungal agar plug covered with mycelium, and spore suspension (Baldrian, 2004; Mata et al., 2005; Flores et al., 2010). However, these ways are difficult to inoculate a certain amount of mycelium. Likewise, the inoculum size ratios in this experiment do not represent the exact mycelium amount inoculated to culture, because the mycelium density of each strain is different on GPA. But the results from this work clearly showed that the inoculum amount ratio of two strains plays an important role in laccase production during co-culturing.

\subsection{Effect of the infection time of T. longibrachiatum on laccase production}

The effects of infection time of T. longibrachiatum to T. rubrum LKY-7 culture on laccase production at the inoculum size ratio of 0.5 to 1 and 1 to 1 were evaluated. As shown in Fig. 2A, when infected with T. longibrachiatum after 1 day of cultivation at the ratio of 0.5 to 1 , the laccase productions by $T$. rubrum LKY-7 were significantly suppressed and limited. Maximal laccase activity was achieved from the $T$. rubrum LKY-7 culture infected after 3 days at the ratio

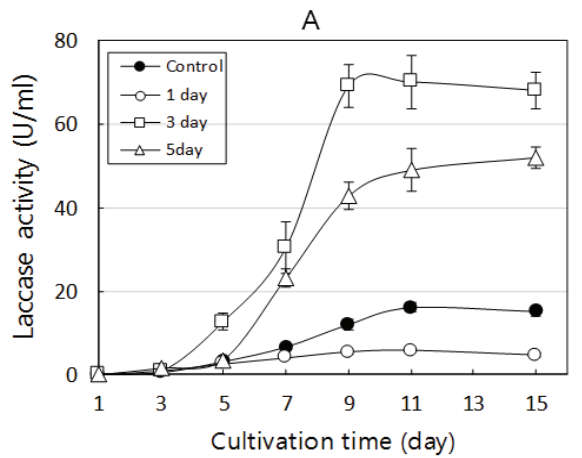

of $0.5: 1$, yielding $70.2 \mathrm{U} / \mathrm{ml}$ of laccase activity on day 11 of cultivation. And, although the laccase activity increased to some extent when infected after 5 days, it was not as high as when infected after 3 days. In contrast, the laccase activities of T. rubrum LKY-7 were almost inhibited when infected after 1 day at the ratio of 1 to 1 (Fig. 2B). However, the laccase activity of culture when infected after 5 days at the ratio of 1 to 1 increased gradually until day 7 of cultivation, then increased rapidly until the end of cultivation. And the laccase activities of $T$. rubrum LKY-7 culture when infected after 5 days cultivation were higher than those of the culture infected after 3 days cultivation at the ratio of 1 to 1 . These results showed the importance of the growth balance for laccase production in coculture. That is, it means that for the enhancement of laccase production, T. rubrum LKY-7 should be precultured enough to gain a competitive advantage in co-culture, as the infection extent of T. longibrachiatum is high (the ratio of 1 to 1 ). Likewise, Cupul et al. (2014) showed that the time at which T. maxima is inoculated with $P$. carneus has a significant effect on laccase activity, and the best time for inoculation with $P$. carneus is $3 \mathrm{~d}$ after T. maxima was established.

The stability of laccase activity is as important as laccase production for industrial and environmental applications. The stability of laccase activity in co-

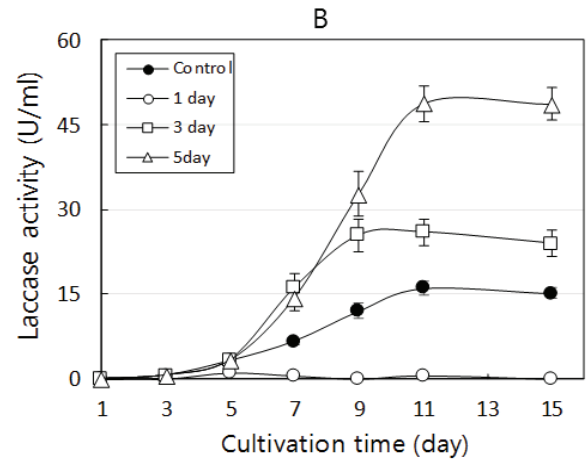

Fig. 2. Effect of the infection time of T. longibrachiatum on laccase production by T. rubrum LKY-7. A, Inoculum size ratio of 0.5 to 1 ; $B$, Inoculum size ratio of 1 to 1 . 
culture was improved with compared to control, rather showing the increase of laccase activities at day 12 of cultivation. Especially, in the co-culture of $T$. rubrum LKY-7 with $T$. longibrachiatum, the maximal laccase activity was remained almost constant until day 15 of cultivation (data not shown). These results are in agreement with the study of Flores et al. (2010), who has shown that the stability of laccase activity is improved during Trichoderma-Pleurotus interaction yielding twice the activity if compared with control treatments (Trichoderma or Pleurotus alone). Also, Zhang et al. (2006) reported that the laccase activity decreases sharply in chemical induction within 2-3 days after peak, whereas it maintains $60-70 \%$ of its highest activity for at least 20 days during the co-cultivation process of Trametes sp. and Trichoderma strain.

Generally, it has well known that the increase of laccase activity by basidiomycetes in co-culture could be a response against the attack of Trichoderma (Boddy, 2000). However, the results from this experiment showed that the effect of infection extent and infection time on laccase production should be considered in its aspects of the growth ability of $T$. longibrachiatum in co-culture. That is, the increase of laccase activity in co-culture was seemed to be related to the competitive ability of $T$. rubrum LKY-7 against $T$. longibrachiatum. In addition, it suggested that for laccase production, the infection extent and the infection time in co-culture should be adjusted so that T. rubrum LKY-7 can be grown up in advance to a certain level, before inoculating T. longibrachiatum.

\subsection{Effects of the culture filtrate and the autoclaved mycelium of T. Iongibrachiatum on laccase production}

The addition of culture filtrate or autoclaved mycelium of T. longibrachiatum to T. rubrum LKY-7 culture did not significantly enhance laccase production.

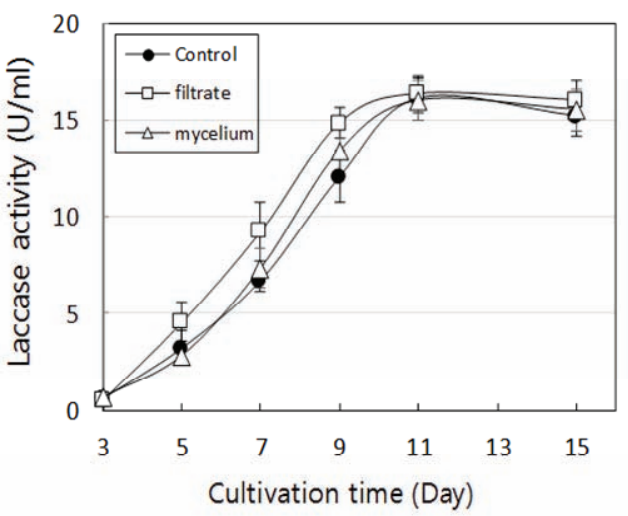

Fig. 3. Effects of the culture filtrate and the autoclaved mycelium of $T$. longibrachiatum on laccase production by $T$. rubrum LKY-7.

As shown in Fig. 3, although the laccase activity of T. rubrum LKY-7 culture increased to some extent with the addition of culture filtrate, it was quite a low level, when compared with the maximal laccase activity obtained from co-culture. And the addition of autoclaved mycelium appeared to be little effect on the enhancement of laccase production. This suggested that laccase production in co-culture was enhanced mainly by the live and active mycelium of $T$. longibrachiatum. These results are in agreement with Baldrian (2004), who reported that the addition of sterilized T. harzianum culture homogenate did not significantly affect the enzyme activity in the decolorization of RBBR. Also, the same results (no laccase induction) were obtained when sterile-filtered culture liquid from $T$. versicolor $\mathrm{x} T$. harzianum cultures were added to the flasks with T. versicolor. On the other hand, it has been proposed that Trichoderma sp. and/or its metabolites stimulate the laccase production of ligninolytic fungi (Savoie et al., 1998; 2001; Savoie and Mata, 1999, Hatvani et al., 2002). Mata et al. (2005) demonstrated that a significant increase in laccase activity was observed when cultures of $P$. pulmonarius were incubated with the Trichoderma lytic enzyme, representing more than an eight-fold increase in production compared to the 
P. pulmonarius control. Also, it has been suggested that extracellular laccase production of $L$. edodes may be due to cell wall degrading enzymes produced by Trichoderma (Savoie and Mata, 2003). In contrast, Zhang et al. (2006) suggested that some thermalresistant compounds, but not lyases, in the extracellular metabolites of Trichoderma sp. ZH1 can singly act as signals to induce laccase production of Trametes sp. AH28-2 strongly. This implies that recognition of the host (resulting in a direct attack to basidiomycetes) by Trichoderma and its production of non-enzymatic metabolites are both factors worth considering the potential for increased laccase production by $P$. ostreatus or A. bisporus (Flores et al., 2009). However, the results from this work showed that the live and active mycelium of $T$. longibrachiatum is more effective on the enhancement of laccase production by $T$. rubrum LKY-7 in co-culture than its culture filtrate or autoclaved mycelium.

\subsection{Laccase isozymes in native PAGE}

The native PAGE showed that $T$. rubrum LKY-7 produced two major bands (band 1 and 2) and two minor bands (band 3 and 4) of laccase activities (Fig. 4). Mata et al.(2005) observed two patterns of main bands formed by Pleurotus strain according to the kind of culture media with or without Trichoderma. Flores et al. (2010) reported that Pleurotus ostreatus produces two laccase band, which are always present throughout the cultivation time, while laccase isoforms pattern changed after the infection of $P$. ostreatus cultures with T. viride. In this work, no inducible laccase isozyme or laccase modification was observed both on GPA and GPB. It showed that when co-cultured with $T$. longibrachiatum, the intensity of laccase band 4 increases both on GPA and GPB in comparison with that of mono-culture of $T$. rubrum LKY-7 on GPB. From these results, it could be supposed that laccase

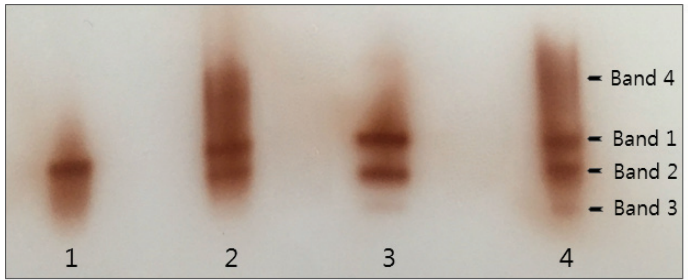

Fig. 4. Detection of laccase isozymes by native PAGE. Lane 1, self-paired culture of LKY-7 in GPA Lane 2, co-culture in GPA

Lane 3, mono-culture of LKY-7 in GPB

Lane 4, co-culture in GPB.

band 4 was related to the enhancement of laccase production in co-cultivation on GPA and GPB. Interestingly, only one laccase band was detected in self-paired culture of T. rubrum LKY-7 on GPA, while all laccase isoforms were observed in mono-culture of T. rubrum LKY-7 on GPB. Mata et al. (2005) reported that the presence of Trichoderma does not seem to induce new laccase isoforms, but rather, it only seems to stimulate the overproduction of this enzyme. Flores et al. (2009) have shown that while two laccases were observed in liquid co-culture, only one isoform was observed during confrontation of $P$. ostreatus with $T$. viride in solid media. And they added that fungal interaction in solid media, although yielding only one laccase, stimulates the production of a highly reactive isoform. Unlike those results, the expression of only one laccase band could be involved in the stability of laccase activity on GPA. That is, the laccase activity decreased significantly at day 12 of cultivation in self-paired culture on GPA (Table 1), at which only one laccase band was detected. On the other hand, the laccase activity remained relatively stable until the end of cultivation in mono-culture in GPB (Fig. 1), at which laccase isoforms pattern did not change. Accordingly, it could be considered that some laccase isozymes in self-paired culture on GPA were removed by autolysis, resulted in the significant decrease of laccase activity at the latter period of cultivation. 


\section{CONCLUSION}

The co-culture of $T$. rubrum LKY-7 with $T$. longibrachiatum was found to have a potential for laccase production and for laccase stability in GPB (liquid medium). A significant increase of laccase activity was observed when T. rubrum LKY-7 was cocultured with $T$. longibrachiatum in GPB, yielding an increase of more than 5 times in laccase activity, as compared with control. The laccase production by $T$. rubrum LKY-7 during co-culturing was greatly influenced by the infection extent and the infection time of $T$. longibrachiatum. It showed that for the enhancement of laccase production, the infection extent and the infection time of $T$. longibrachiatum in co-culture should be adjusted so that T. rubrum LKY-7 can grow in advance to a certain level, before inoculating $T$. longibrachiatum. Maximal laccase activity was obtained when the T. rubrum LKY-7 culture was infected by T. longibrachiatum after 3 days of cultivation at 0.5 to 1 of inoculum size ratio of $T$. longibrachiatum to T. rubrum LKY-7 in GPB. The addition of culture filtrate or autoclaved mycelium of $T$. longibrachiatum into $T$. rubrum LKY-7 culture did not significantly enhance the laccase production by T. rubrum LKY-7 when compared with control (mono-culture of T. rubrum LKY-7). In native PAGE, no inducible laccase isozyme was observed in co-cultures in comparison with the mono-culture of $T$. rubrum LKY-7, only showing the increase of intensity of laccase band both on GPA and GPB. On the other hand, only one laccase band was detected in self-paired culture of T. rubrum LKY-7 on GPA, while all laccase isoforms were observed in mono-culture of $T$. rubrum LKY-7 on GPB.

\section{ACKNOWLEDGMENT}

This paper was supported by Sunchon National University Research Fund in 2018.

\section{REFERENCES}

An, J.E., Lee, S.Y., Ryu, S.H., Kim, M.K. 2018. Transformation of terpene synthase from Polyporus brumalis in Pichia pastoris for recombinant enzyme solution. Journal of the Korean Wood Science and Technology 46(4): 415-422.

Baldrian, P. 2004. Increase of laccase activity during interspecific interaction of white rot fungi. FEMS Microbiology Ecology 50: 245-253.

Baldrian, P., Gabriel, J. 2002. Copper and cadmium increase laccase activity in Pleurotus ostreatus. FEMS Microbiology Letters 206: 69-74.

Boddy, L. 2000. Interspecific combative interactions between wood-decaying basidiomycetes. FEMS Microbiology Ecology 31: 185-194.

Chi, Y., Hataka, A., Maijala, P. 2007. Can co-culturing of two white rot fungi increase lignin degradation and the production of lignin degrading enzymes? International Biodeterioration and Biodegradation 59: 32-39.

Cho, M.K., Ryu, S.H., Kim, M.K. 2012. Changes in activities of lignin degrading enzymes and lignin content during degradation of wood chips by Polyporus brumalis. Journal of the Korean Wood Science and Technology 40(6): 424-430.

Choi, Y.J., Shin, Y.S., Cho, N.S. 2006. Screening of wood-rot fungi based on RBBR decolorization and its laccase activity. Journal of the Korean Wood Science and Technology 34(4): 46-53.

Cupul, W.C., Abarca, G.H., Carrera, D.M., Vázquez, R.R. 2014. Enhancement of ligninolytic enzyme activities in a Trametes maxima-Paecilomyces carneus co-culture : Key factors revealed after screening using a Plackett-Burman experimental design. Eletronic Journal of Biotechnology 17: 13-25.

Eggert, C., Temp, U., Eriksson, K-EL. 1996. The ligninolytic system of the white rot fungus 
Enhancement of Laccase Production from Wood-Rotting Fungus by Co-Culture with Trichoderma longibrachiatum

Pycnoporus cinnabarinus: purificaion and characterization of the laccase. Applied and Environmental Microbiology 62: 1151-1158.

Flores, C., Casasanero, R., Trejo-Hernández, M.R., Galindo, E., Serrano-Caqrreón, L. 2010. Production of laccases by Pleurotus ostreatus in submerged fermentation in co-culture with Trichoderma viride. Journal of Applied Microbiology 108: 810-817.

Flores, C., Vidal, C., Trejo-Hernández, M.R., Galindo, E., Serrano-Caqrreón, L. 2009. Selection of Trichoderma strains capable of increasing laccase production by Pleurotus ostreatus and Agaricus bisporus in dual cultures. Jounal of Applied Microbiology 106: 249-257.

Hatvani, N., Kredics, L., Antal, Z., Mécs, I. 2002. Changes in activity of extracellular enzymes in dual cultures of Lentinula edodes and mycoparasitic Trichoderma strains. Journal of Applied Microbiology 92: 415-423.

Hiscox, J., Balddrian, P., Rogers, H.J., Boddy, L. 2010. Changes in oxidative enzyme activity during interspecific mycelial interactions involving the white-rot fungus Trametes versicolor. Fungal genetics and Biology 47: 562-571.

Hong, C.H., Kim, H.Y., Jang, S.K., Choi, I.G. 2013. Screening of outstanding white rot fungi for biodegradation of organosolv lignin by decolorization of Remazol Brilliant Blue $\mathrm{R}$ and ligninolytic enzymes systems. Journal of the Korean Wood Science and Technology 41(1): 19-32

Iakovlev, A., Stenlid, J. 2000. Spatiotemporal patterns of laccase activity in interacting mycelia of wood-decaying basidiomycete fungi. Microbial Ecology 39: 236-245.

Jarosz-Wilkolazka, A., Malarczyk, E., Leonowicz, A., Cho, N.S. 2004. Effect of cadium ions on the activity of fungal laccase and its decolorization of dye, RBBR. Journal of the Korean Wood Science and Technology 32(6): 14-22.
Jung, H.C., Feng, X, Li., K. 2001. Purification and characterization of laccase from wood-degrading fungus Trichophyton rubrum LKY-7. Enzyme and Microbial Technology 30: 161-168.

Lee, K.Y., Park, S.G., Park, I.H., Kim, J.S., Park, M.S., Jung, H.C. 2017. Mycelial response and ligninolytic enzyme production during interspecific interaction among wood-rotting fungi. Journal of Mushrooms 15(4): 168-177.

Lee, S.Y., Ryu, S.H., Choi, I.G., Kim, M.K. 2016. Biosynthesis of Eudesmane-type Sesquiterpenoids by the Wood-rotting Fungus, Polyporus brumalis, on Specific Medium, including Inorganic Magnesium Source 1. Journal of the Korean Wood Science and Technology 44(2): 253-263.

Mata, G., Murrieta Hernández, D.M., Iglesias Andreu, L.G. 2005. Changes in lignocellulolytic enzyme activities in six Pleurotus spp. strains cultivated on coffee pulp in confrontation with Trichoderma spp. World Journal of Microbiology and Biotechnology 21: 143-150.

Minussi, R.C., Pastore, G.M., Duran, N. 2002. Potential applications of laccase in the food industry. Trends in Food Science and Technology 13: 205-216.

Pooja, U., Rahul, S., Pavan-Kumar, A. 2016. Bioprospecting and biotechnological applications of fungal laccase. 3 Biotech 6: 15-26.

Savoie, J.M., Mata, G. 1999. The antagonistic action of Trichoderma sp. hyphae to Lentinula edodes hyphae changes lignocellulolytic activities during cultivation in wheat straw. World Journal of Microbiology and Biotechnology 15: 369-373.

Savoie, J.M., Mata, G., Billette, C. 1998. Extracellular laccase production during hyphal interactions between Trichoderma sp. and Shiitake, Lentinula edodes. Applied Microbiology and Biotechnology 49: 589-593.

Savoie, J.M., Mata, G., Mamoun, M. 2001. Variability in brown line formation and extracellular laccase 
production during interaction between white-rot basidiomycetes and Trichoderma harzianum biotype Th2. Mycologia 93: 243-248.

Téllez-Téllez, M., Díaz-Godínez, G., Sánchez, C. 2003. Physiology of colony of Pleurotus pulmonarius grown on medium overlaid with a cellophane membrane. Applied Microbiology and Biotechnology 63: 212-216.

Thurston, C.F. 1994. The structure and function of fungal laccase. Microbiology 140: 19-26.

Valle, J.S., Vandenberghe, L.P.S., Oliveira1, A.C.C., Tavaresl, M.F., Linde, G.A., Colauto, N.B., Socco, C.R. 2015. Effect of different compounds on the induction of laccase production by Agaricus blazei. Genetics and Molecular Research 14: 1588215891.
Velázquez-Cedeño, M., Farnet, A.M., Billette, C., Mata, G., Savoie, J.M. 2007. Interspecific interactions with Trichoderma longibrachiatum induce Pleurotus ostreatus defence reactions based on the production of laccase isozymes. Biotechnology Letters 29: 1583-1590.

Yadav, B. 2018. Induction of laccase in fungus, Cyathus stercoreus using some aromatic inducers. Journal of Applied and Natural Science 10(1): 445-447.

Zhang, H., Hong, Y.Z., Xiao, Y.Z., Yuan, J., Tu, X.M., Zhang, X.Q. 2006. Efficient production of laccase by Trametes sp. AH28-2 in co-cultivation with Trichoderma strain. Applied Microbiology and Biotechnology 73: 89-94. 